\title{
Personalized alignment in total knee arthroplasty: current concepts
}

\author{
Sébastien Lustig ${ }^{1,2}$, Elliot Sappey-Marinier ${ }^{1}$, Camdon Fary ${ }^{3,4}$, Elvire Servien ${ }^{1,5}$, Sébastien Parratte ${ }^{6,7}$, \\ and Cécile Batailler ${ }^{1,2, *}$
}

${ }^{1}$ Orthopaedics Surgery and Sports Medicine Department, FIFA Medical Center of Excellence, Croix-Rousse Hospital, Lyon University Hospital, 69004 Lyon, France

2 Univ Lyon, Claude Bernard Lyon 1 University, IFSTTAR, LBMC UMR_T9406, 69622 Lyon, France

3 Department of Orthopaedic Surgery, Western Health, 3002 Melbourne, Australia

4 Australian Institute for Musculoskeletal Science (AIMSS), The University of Melbourne and Western Health, 3002 St. Albans, VIC, Australia

5 LIBM - EA 7424, Interuniversity Laboratory of Biology of Mobility, Claude Bernard Lyon 1 University, 69003 Lyon, France

${ }^{6}$ International Knee and Joint Centre, 46705 Abu Dhabi, United Arab Emirates

7 Institute for Locomotion, Aix-Marseille University, 13009 Marseille, France

Received 17 February 2021, Accepted 3 March 2021, Published online 26 March 2021

\begin{abstract}
Traditionally in total knee arthroplasty (TKA), a post-operative neutral alignment was the gold standard. This principle has been contested as functional outcomes were found to be inconsistent. Analysis of limb alignment in the non-osteoarthritic population reveals variations from neutral alignment and consideration of a personalized or patient-specific alignment in TKA is challenging previous concepts. The aim of this review was to clarify the variations of current personalized alignments and to report their results. Current personalized approaches of alignment reported are: kinematic, inverse kinematic, restricted kinematic, and functional. The principle of "kinematic alignment" is knee resurfacing with restitution of pre-arthritic anatomy. The aim is to resurface the femur maintaining the native femoral joint line obliquity. The flexion and extension gaps are balanced with the tibial resection. The principle of the "inverse kinematic alignment" is to resurface the tibia with similar medial and lateral bone resections in order to keep the native tibial joint line obliquity. Gap balancing is performed by adjusting the femoral resections. To avoid reproducing extreme anatomical alignments there is "restricted kinematic alignment" which is a compromise between mechanical alignment and true kinematic alignment with a defined safe zone of alignment. Finally, there is the concept of "functional alignment" which is an evolution of kinematic alignment as enabling technology has progressed. This is obtained by manipulating alignment, bone resections, soft tissue releases, and/or implant positioning with a robotic-assisted system to optimize TKA function for a patient's specific alignment, bone morphology, and soft tissue envelope. The aim of personalizing alignment is to restore native knee kinematics and improve functional outcomes after TKA. A long-term follow-up remains crucial to assess both outcomes and implant survivorship of these current concepts.
\end{abstract}

Key words: Total knee arthroplasty, Personalized alignment, Kinematic alignment, Restricted alignment, Functional alignment, Implant survivorship.

\section{Nomenclature}

CT scan Computerized Tomography scan

HKA Hip Knee Ankle angle

KA Kinematic Alignment

LDFA Lateral Distal Femoral Angle

LTR Lateral Tibial Resection

MA Mechanical Alignment

mFA Mechanical Femoral Axis

MPTA Medial Proximal Tibial Angle

mTA Mechanical Tibial Axis

\footnotetext{
*Corresponding author: cecile-batailler@hotmail.fr
}

$\begin{array}{ll}\text { MTR } & \text { Medial Tibial Resection } \\ \text { OKS } & \text { Oxford Knee Score } \\ \text { PCA } & \text { Posterior Condylar Axis } \\ \text { STE } & \text { Surgical Trans Epicondylar axis } \\ \text { TKA } & \text { Total Knee Arthroplasty } \\ \text { TJL } & \text { Tibial Joint Line }\end{array}$

\section{Introduction}

Traditionally in total knee arthroplasty (TKA), a postoperative neutral alignment was a standard principle [1-3]. To obtain a mechanical alignment the femoral and tibial 
components are positioned at $90^{\circ}$ to the tibial and femoral mechanical axis. This alignment philosophy for knee arthroplasty was driven by equalizing load on the implant to decrease wear and loosening rather than restoring normal knee kinematics and function. Mechanical Alignment (MA) in TKA has demonstrated good long-term implant survival [2, 4, 5]. However, functional outcomes of the TKA are inconsistent. Bonnin et al. found $75 \%-89 \%$ of patients with TKA reported significant discomfort [6]. Discomfort during activities of daily living is a significant cause of patient's dissatisfaction after TKA [6-8].

Several recent studies have described limb alignment in non-osteoarthritic and osteoarthritic populations. A systematic review by Moser et al. reported that the mean hip knee ankle angle (HKA) ranged from 176.7 to $180.7^{\circ}$ in a native nonosteoarthritic knee [9]. The majority of studies in the review (12-15) did not report a neutral native limb alignment of $180^{\circ}$, apart from Hovinga and Lerner [10] or Khattak et al. [11]. The coronal alignment variability in non-osteoarthritic knees raises the question of a limb alignment of $180^{\circ}$ is "normal". This alignment could be not the target in TKA for all patients. Hess et al. in a second paper reviewed femorotibial alignment in osteoarthritic knees and concluded there were a large variation in overall coronal limb alignment as well as isolated tibial and femoral coronal alignments [12]. This observation continues to fuel the discussion and classification of limb alignment. In an asymptomatic Cohort of 250 adults, Bellemans et al. described a neutral alignment as $180^{\circ} \pm 3^{\circ}$, constitutional varus inferior to $177^{\circ}$, and constitutional valgus superior to $183^{\circ}$ [13]. Hirschmann et al. in more recent studies further classified the HKA alignment to include the femoral and the tibial mechanical angles (FMA and TMA, respectively) [14-16]. This classification is more useful and is an explanation of how current concepts of alignment variations in both femoral and tibial cuts will affect the final alignment.

As the concept of MA was questioned in the $1980 \mathrm{~s}$ anatomical alignment was described by Krackow and Hungerford with the aim to improve functionality by closer reproducing the native knee alignment [1, 17], but the alignment was similar for all and not personalized. This lead to the development of several concepts of personalized alignment: kinematic, inverse kinematic, restricted kinematic, and functional. The distinction between these different concepts of alignment is sometimes difficult to interpret and reporting inconsistent in the literature.

The goal of this current concepts paper is to clarify the different types of current personalized alignments, summarize their main principles and report their results.

\section{Kinematic alignment}

\section{Principles}

Kinematic alignment (KA), described by Howell et al. in 2006, is an "individualized" or patient-specific technique [18]. The aim of KA is knee resurfacing with restitution of the pre-arthritic anatomy and preservation of the soft-tissue envelope. In this technique the knee is represented in three kinematic axis with respect to the joint lines of the posterior and distal femur (Figure 1): one transverse axis in the femur about which the tibia extends and flexes, one about which the patella extends and flexes and one longitudinal axis about which the tibia externally and internally rotates on the femur. All three axes are either parallel or perpendicular to the joint lines [19]. By resurfacing the femorotibial joint, the KA technique aims to co-align the axes and joint lines of implants with the three "kinematic" axes and joint lines of the native joint. The surgeon resurfaces the femur maintaining the pre-arthritic femoral joint line obliquity, and adjusts the extension and flexion gaps with the resection of the proximal tibia. Sometimes, KA involves complex algorithms to balance the extension and flexion gaps [20]. The tibial compensation can result in more oblique tibial varus resections with an increased medial tibial cut compared to MA.

Tibial and femoral resection thicknesses are validated with caliper measures and must match the thickness of the implants after compensating for saw cut and wear. It restores pre-arthritic ligament lengthening, does not create gap imbalance, minimizes the need for release [21-24]. Howell does not place restrictions on the patient's anatomy or final correction. For this reason, KA requires an accurate surgical technique and can be performed by multiple methods: conventional instrumentation, computer navigation, personalized instruments, or robotic-assisted.

\section{Surgical technique}

KA implantation is usually a measured resection technique with the femur first (Figure 2b). Initially, the surgeon must estimate the individual physiological knee laxity throughout the range of motion of the knee and the amount of bone loss. The first cut is the distal femoral cut which is parallel to the joint line after correcting for the estimated bone loss. The posterior femoral cut is then performed parallel to the posterior condylar plane (usually no wear posteriorly). Resection of bone (corrected for wear) from the posterior and distal femur is equal in thickness to the femoral implant condyle which kinematically aligns the femoral implant. The surgeon then cuts the tibia parallel to the joint line. The tibial resected bone (corrected for wear) is equal in thickness to the tibial component will kinematically align the tibial component [19].

The surgeon must always measure each bone resection with a caliper. The thickness of the bone cut is calculated by deducting $1 \mathrm{~mm}$ from the component thickness for the saw cut thickness and by estimating the amount of articular surface wear. The cartilage thickness is frequently almost $2 \mathrm{~mm}$ on the distal and posterior parts of the femoral condyles.

During the trials, if there is a femorotibial soft-tissue imbalance (tightness, excessive laxity) and the soft-tissue envelope remains intact (no release/deficiency), the proximal tibia should be recut to compensate. Kinematic femoral component implantation is relatively straightforward and highly reproducible compared to a kinematic tibial cut and component insertion. A common technique for this method is to use personalized (patient-specific) cutting guides that enable additional degrees of slope/valgus/varus. In summary, the ligament balancing is performed by the bone cuts and adjusted as required by the tibial cut. This results in two important limitations that can occur with KA and have led to the development of restricted $\mathrm{KA}$ and inverse KA which is discussed later. 

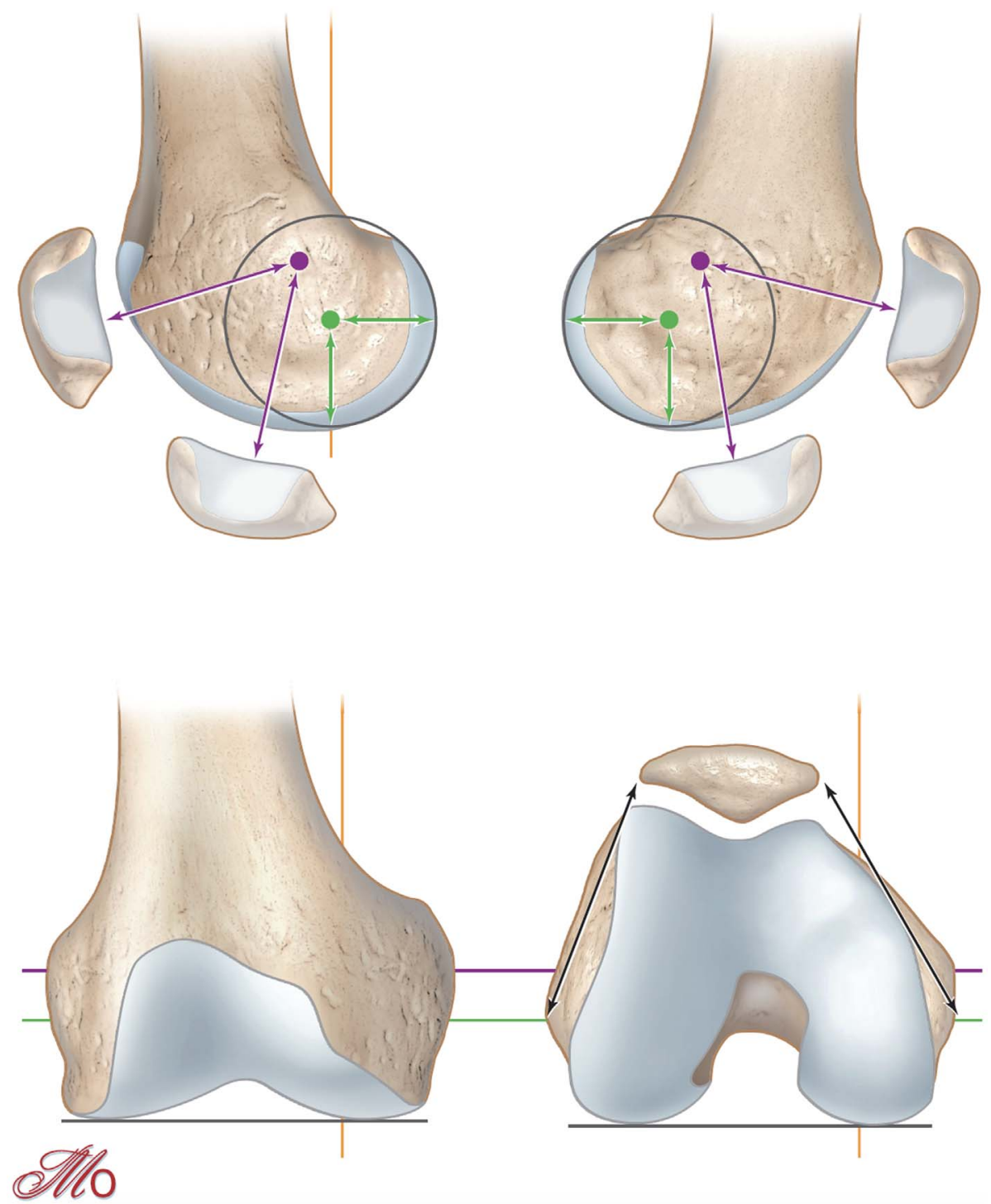

Figure 1. The femoral transverse axis about which the tibia extends and flexes is the most distal and posterior (Green line). The femoral transverse axis about which the patella extends and flexes is more proximal and anterior (Violet line). The longitudinal axis about which the tibia externally and internally rotates on the femur passes through the medial femorotibial compartment (Yellow line). All three axes are either perpendicular or parallel to the joint lines (Blackline).

\section{Results}

Sappey-Marinier et al. performed a systematic review of the clinical and radiological outcomes after TKA with KA versus with MA at 2 years of follow-up [25]. They reported that four of five prospective randomized controlled trial studies did not find any difference between the two groups (MA or KA) for all scores [26-29]. One study reported that kinematically aligned TKA had significantly better scores for a range of motion, function, pain than those who underwent mechanically aligned TKA [30]. Young et al. [26] found no difference between kinematic alignment $(n=49)$ and mechanical alignment $(n=50)$ in Oxford Knee Score (OKS) $(42 \pm 6$ and $41 \pm 6$, respectively) at 2-years follow-up. A randomized controlled trial by Dossett et al. [30] revealed a significant difference $(p=0.005)$ with KA outcomes $(n=44)$ greater than
MA $(n=44)$ in OKS $(40 \pm 10.2$ and $33 \pm 11.1$, resp. $)$ at 2 -years follow up. Of note, $90 \%$ of knees in the latter study were preoperatively in varus alignment and at 24 months there was no differences concerning the complication and revision rates, postoperative gait analysis, and tibial component migration.

Shelton et al. have assessed the functional outcomes and satisfaction rate of patients treated with a kinematically aligned TKA that had a contralateral MA TKA [31]. 83\% of patients were satisfied with the mechanically aligned TKA when they were treated with the kinematically aligned TKA and $92 \%$ were satisfied with the KA TKA at the last follow-up. The median forgotten joint score (FJS) for KA TKA was higher than MA TKA by a significant difference of 15 points $(p=0.006)$. $56 \%$ favored the KA TKA, $8 \%$ favored the mechanically aligned TKA, and $36 \%$ rated both knees the same $(p<0.001) .74 \%$ of patients favored the recovery of the 

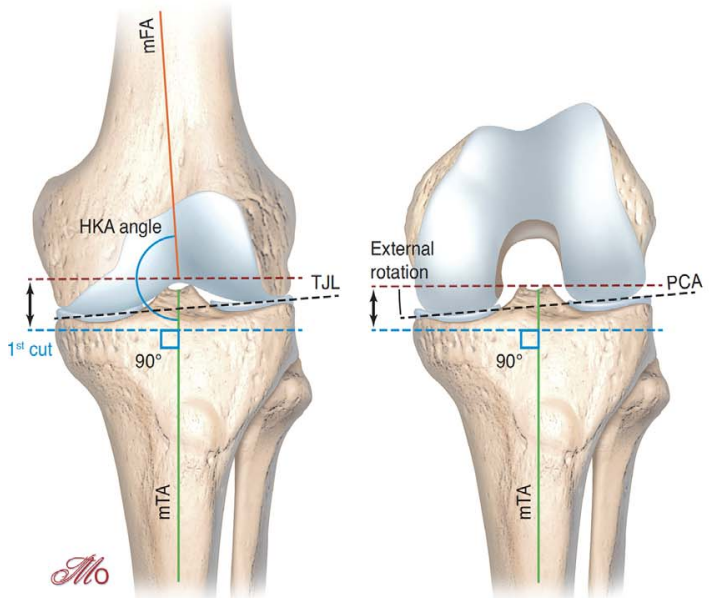

(a)

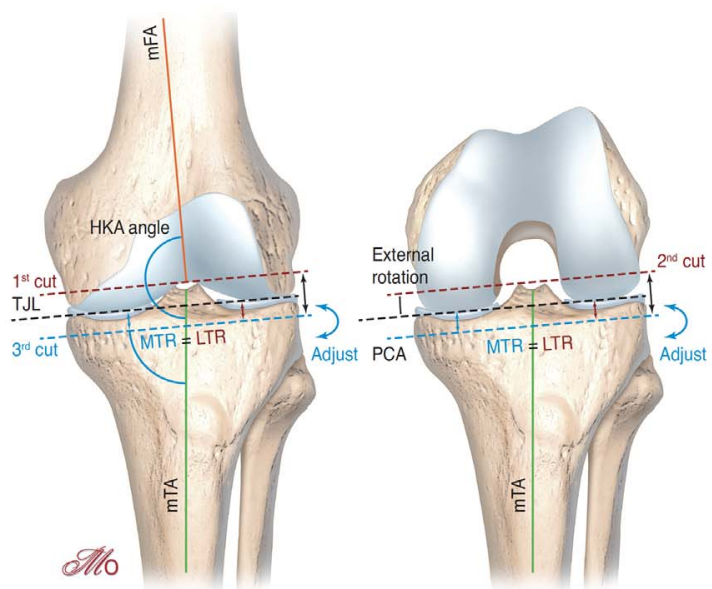

(b)

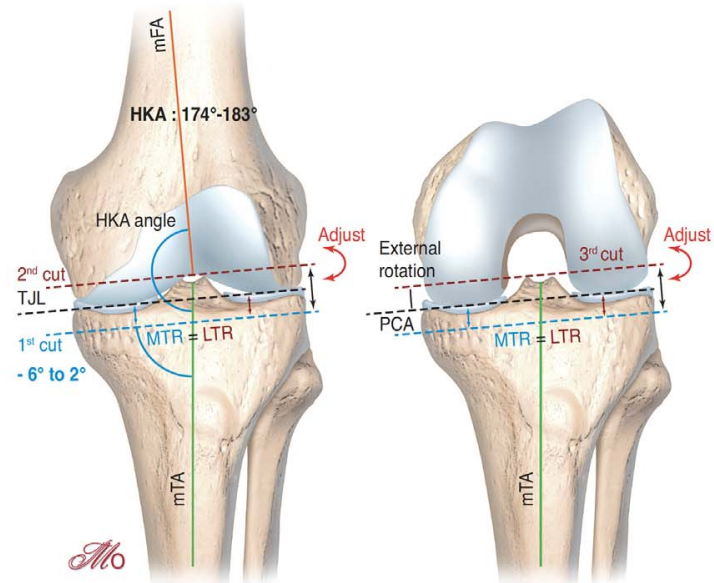

(c)

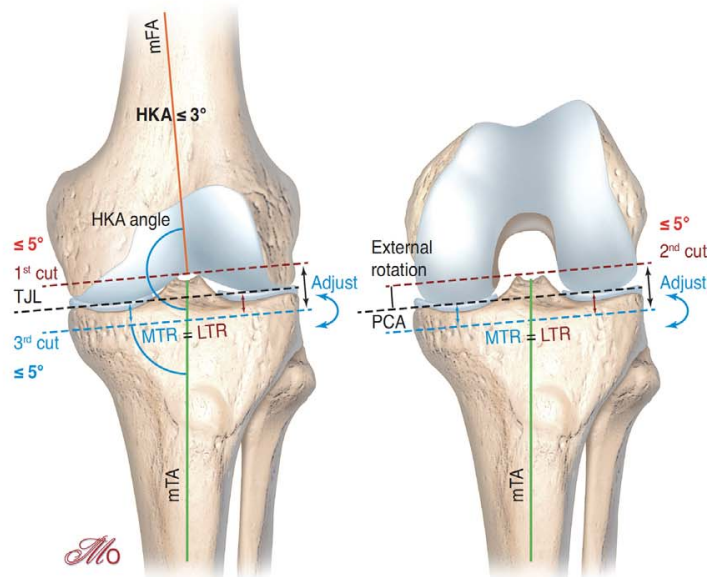

(d)

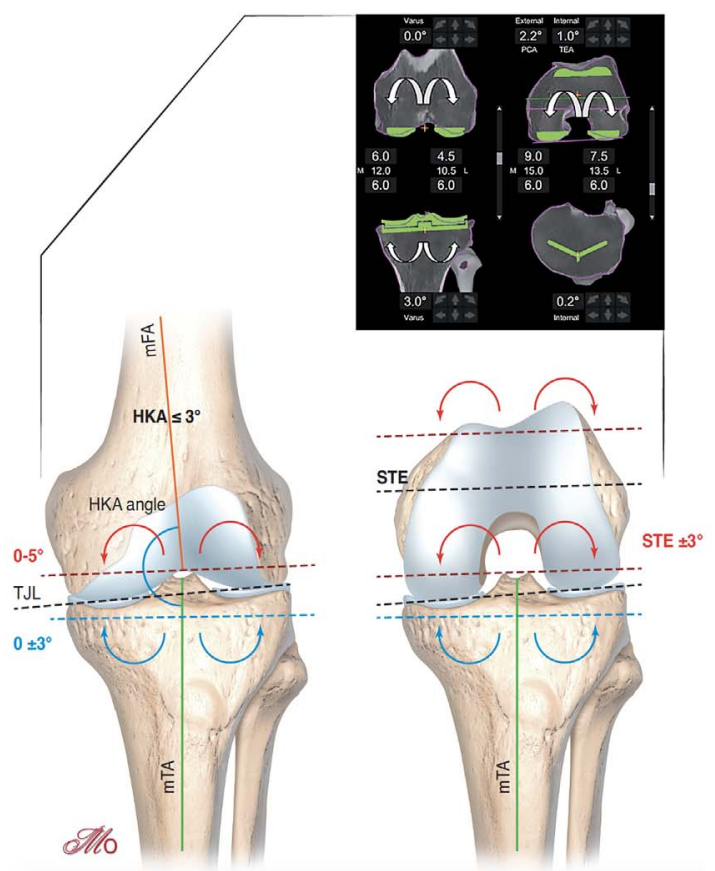

(e)

Figure 2. (a) Mechanical alignment, (b) kinematic alignment, (c) inverse kinematic alignment, (d) restricted kinematic alignment, (e) functional alignment. 
kinematically aligned TKA, $6 \%$ favored the recovery of the mechanically aligned TKA.

A concern with kinematic alignment is the risk of developing aseptic loosening due to the increased varus alignment. Howell et al. reported implant survival of 220 (unrestricted) KA TKA at 10 years of follow-up of $97.5 \%$ for revision for any reason and $98.4 \%$ for aseptic failure [23]. Tibial implant loosening occurred in 1 patient, with a reverse tibial slope. Using MA criteria, the percentage postoperatively aligned in the varus (valgus) outlier range $\left(>3^{\circ}\right)$ was $78 \%(0 \%)$ for the tibial implant, $31 \% 220(5 \%)$ for the femoral implant knee, and $7 \%(21 \%)$ for the HKA (unknown mean varus).

\section{Inverse kinematic alignment}

\section{Principles}

A limitation of correcting ligament balancing with a tibial recut is that the "resurfacing" of the femur is at the expense of adjustment with the tibial cut. Two difficulties can occur if a tibial recut is necessary for ligament rebalance. Firstly, a more oblique and deeper recut will sacrifice medial tibial bone stock. Sappey-Marinier has demonstrated that an increased tibial resection depth is associated with significantly greater laxity in valgus between 30 and $90^{\circ}$ of flexion, particularly with a tibial resection $\geq 14 \mathrm{~mm}$ [32]. Increasing the tibial resection could jeopardize the medial collateral ligament and could complicate TKA revision if required. The risk of early loosening with tibial secondary displacement is increased with a severe varus tibial alignment [33]. The second difficulty concerns gap balancing where an increased tibial recut impacts the flexion and the extension gaps. In the majority of "standard" cases the difference between gaps is small. But in complex cases where the recut may be asymmetrical, it could lead to laxity.

The principle of the "inverse kinematic alignment" is to "resurface" the tibia with similar medial and lateral resections after correcting for wear, maintaining the pre-articular tibial joint line obliquity. The gap balancing is then performed by adjusting the femoral posterior and distal resections (Figure 2c). This technique could avoid tibial over resection and tibiarelated complications postoperatively. This technique has the advantage to manage independently the flexion and extension gaps. However, to perform an inverse KA accurately with conventional instrumentation or patient-specific guides is challenging and complex while a robotic-assisted system enables intraoperative planning of bone resections and gap balancing before the cuts.

\section{Surgical technique}

Winnock de Grave et al. described this new concept and technique with a robotic-assisted system [34]. The tibial implant is positioned first with resection of equal amounts of bone lateral and medial on the tibia, after correcting for bone wear. The goal is to restore the native medial proximal tibial angle, within a safe zone of $84-92^{\circ}$. The tibial slope is determined by the pre-arthritic medial tibial slope. On the femoral side, the femoral implant is positioned to restore the medial joint line height both in flexion and extension. The extension and flexion gaps are balanced by adjusting the posterior and distal femoral resection levels. For the flexion gap, the goal is to achieve with the robotic-assisted system residual laxity of $1-3 \mathrm{~mm}$ in the lateral compartment and 1-2 $\mathrm{mm}$ in the medial compartment. For the extension gap, the goal is to achieve with the roboticassisted system residual laxity of 1-2 $\mathrm{mm}$ in the two compartments. The target for the HKA angle remains in a safe zone between 174 and $183^{\circ}$. Readjustment of the femoral cuts a second time after the first cuts after trialing is difficult with a conventional resection guide. The robotic-assisted system estimates gap balancing prior to the cuts but also to potentially estimate and perform an adjustment and recuts after initial resections and trial.

\section{Results}

Only Winnock de Grave et al. have reported the outcomes of the inverse KA. They found no significant difference in clinical results at 12 months between inverse KA and adjusted MA [34]. They reported a higher rate of satisfaction and significant improvement in postoperative OKS for restricted inverse $\mathrm{KA}$, compared to adjusted MA. Of note, knees with preoperative varus deformity had an apparent improved functional score and satisfaction for restricted inverse KA compared to adjusted MA. No complication or revision was reported in both groups in the short term. However, these early results require further studies with increased patients and longer follow-up.

\section{Restricted kinematic alignment}

\section{Principles}

KA without restriction remains controversial due to the increased stress on the implants as the knee deformity increases and alignment deviates from MA increasing the risk of aseptic loosening. Nakamura et al. with finite element analysis assessed the tibiofemoral contact force in relation to the limb alignment [35]. In the varus knees, KA increases the contact stress on the tibial insert, medial tibial cortex, and bone resection surface. For moderate $\left(10^{\circ}\right)$ and severe $\left(15^{\circ}\right)$ varus knees, the maximum stress in kinematically aligned TKA increased by 24.8 and $32.2 \%$, compared with to mechanically aligned TKA.

To account for the increasing stress, Vendittoli recommended "safe zones" for TKA alignment. He purposed a restricted KA protocol [36]. Advanced osteoarthritic knee anatomy is very variable and to avoid reproducing extreme anatomy, the restricted KA is a hybrid option between MA and KA. The algorithm involves modifications of bone cuts within a "safe range" defined by some criteria: independent femoral and tibial cuts must be within $\pm 5^{\circ}$ of the mechanical axis and the HKA angle must fall within $\pm 3^{\circ}$ of neutral. But the restricted KA technique follows the main technical principle of the KA technique, which is to respect as much as possible the KA of the femoral implant, and adjustment of the coronal limb alignment and joint line obliquity is first performed by adjusting the tibial implant cut. 


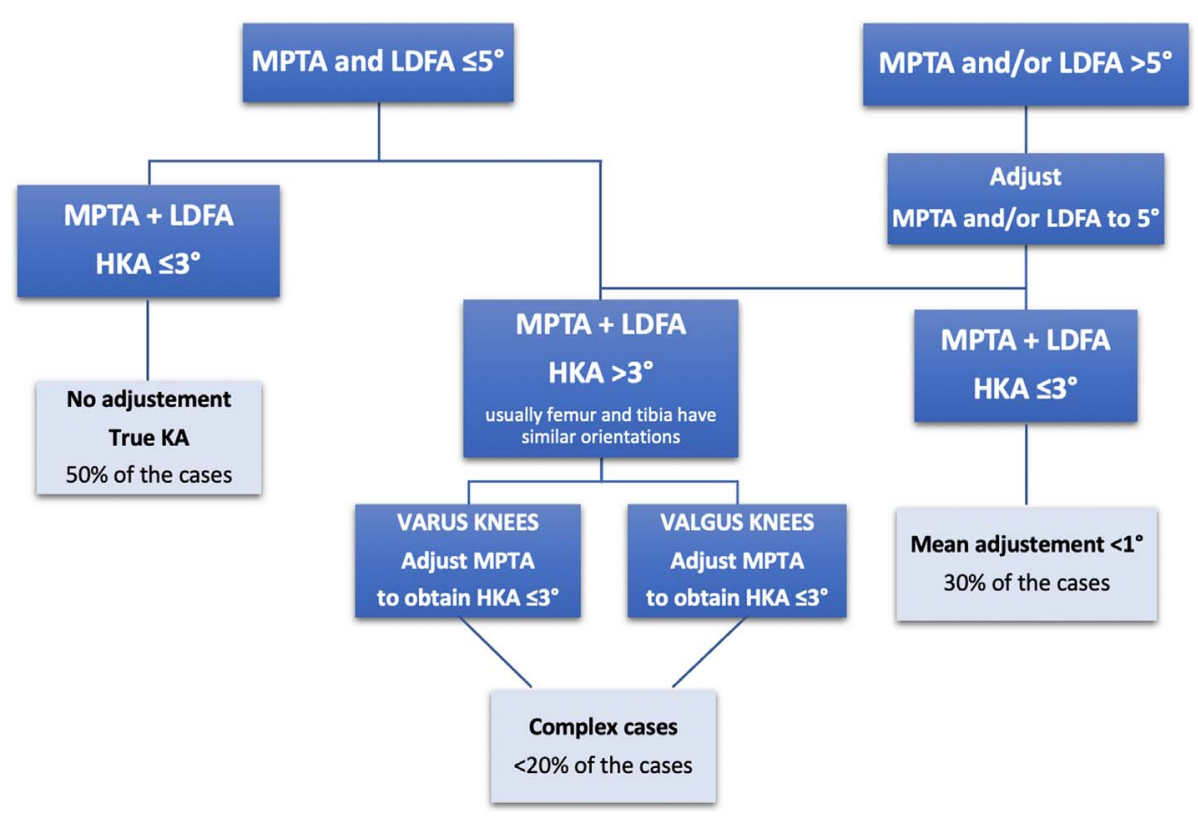

Figure 3. Restricted kinematic alignment protocol.

\section{Surgical technique}

The surgical planning is well described by Vendittoli (Figure 3). There are two situations: either the tibial and femoral mechanical axis are inferior or equal to $5^{\circ}$, or superior to $5^{\circ}$.

In the first case with femoral and tibial axis inferior to $5^{\circ}$, if the femorotibial axis (HKA angle) is equal or inferior to $3^{\circ}$, the surgeon can perform the TKA with a KA technique. If the femorotibial axis is superior to $3^{\circ}$ of varus, the tibial varus will be reduced until the HKA is equal to $3^{\circ}$ of varus. If the femorotibial axis is superior to $3^{\circ}$ of valgus, the tibial varus will be reduced until the HKA is equal to $3^{\circ}$ of valgus.

In cases where the femoral and tibial axis is superior to $5^{\circ}$, the surgeon will correct the tibial and/or the femoral bone cuts to stay within the $5^{\circ}$ limit. This will correct the overall HKA to within $\pm 3^{\circ}$ of neutral. If the patient maintains an HKA superior to $3^{\circ}$, the surgeon will further adjust the tibial cut as in the first situation.

We prefer to modify the tibia to preserve as much as possible the native femoral anatomy and the flexion axis, as in the KA technique. Releases of the ligaments are not needed in cases with anatomic modifications inferior to $3^{\circ}$. In larger corrections, minimal releases can be added (to a much lesser degree compared to MA).

As with the unrestricted KA technique, it is important to measure the bone resections after each cut. Computer navigation and robotic-assisted systems facilitate intraoperative operative adjustment in complex cases.

\section{Results}

Of 2475 TKA cases Almaawi et al. reported 49\% required restricted KA TKA and 51\% unrestricted KA TKA [36]. Blakeney et al. simulated the extension and flexion gaps on 1000 lower limb CT scan according to the restricted KA or MA protocols. An "imbalance" was defined as a difference between lateral and medial gaps. In extension, there were significantly fewer cases having an imbalance $\geq 3 \mathrm{~mm}$ with restricted KA (8.3\%) versus MA (33\%), and $\geq 5 \mathrm{~mm}$ with restricted KA (1.5\%) versus MA (11\%). With restricted KA, the percentage of knees with space imbalances inferior to $3 \mathrm{~mm}$ in both flexion and extension was $92 \%$ versus $63 \%$ with MA with posterior condylar reference and 49\% with MA with trans epicondylar reference [37].

MacDessi et al. have reported encouraging results after TKA with restricted kinematic alignment in a randomized controlled trial [38]. They found that the mean intraoperative intercompartmental pressure difference at $10^{\circ}$ of flexion in the kinematic group was significantly lower than in the mechanical group, using an interoperative pressure sensor. Overall, participants in the kinematic group were more likely to obtain optimal knee balance ( $80 \%$ vs. $35 \%)$.

Currently, no study has assessed the mid- or long-term clinical outcomes after restricted KA TKA.

\section{Functional alignment}

\section{Principles}

Functional alignment has similar aims and was developed for similar reasons as KA [39, 40]. It constitutes an evolution and increased precision of the KA concept. Patient-specific implants and 3D printed cutting blocks were used preoperatively to achieve KA in total knee arthroplasty. Functional alignment is obtained by manipulating alignment, bone resections, fine-tuning component positioning, and/or soft tissue releases at the surgeon's discretion intraoperatively with robotic-assisted systems to achieve balanced extension-flexion gaps and soft tissue tension while maintaining the patient's native alignment. These new and constantly improving technologies enable quantifiable measurement and precision 
Table 1. Surgical parameters for each kind of alignment.

\begin{tabular}{|c|c|c|c|c|c|c|}
\hline & & $\begin{array}{l}\text { Mechanical } \\
\text { alignment }\end{array}$ & $\begin{array}{l}\text { Kinematic } \\
\text { alignment }\end{array}$ & $\begin{array}{l}\text { Inverse kinematic } \\
\text { alignment }\end{array}$ & $\begin{array}{l}\text { Restricted } \\
\text { alignment }\end{array}$ & $\begin{array}{l}\text { Functional } \\
\text { alignment }\end{array}$ \\
\hline \multirow[t]{6}{*}{$\begin{array}{r}\text { Femoral } \\
\text { component }\end{array}$} & Flexion & $\begin{array}{l}\text { Follows distal } \\
\text { femoral bowing }\end{array}$ & $\begin{array}{l}\text { Follows distal } \\
\text { femoral bowing }\end{array}$ & $\begin{array}{l}\text { Follows distal } \\
\text { femoral bowing }\end{array}$ & $\begin{array}{l}\text { Follows distal } \\
\text { femoral bowing }\end{array}$ & $\begin{array}{l}\text { Follows distal } \\
\text { femoral bowing }\end{array}$ \\
\hline & & $\begin{array}{l}\text { Target: } 0 \text { to } 5^{\circ} \text { of } \\
\quad \text { flexion }\end{array}$ & Target: $2 \pm 3^{\circ}$ & Target: $2 \pm 3^{\circ}$ & Target: $2 \pm 3^{\circ}$ & $\begin{array}{l}\text { Target: } 0 \text { to } 5^{\circ} \text { of } \\
\quad \text { flexion }\end{array}$ \\
\hline & Distal cut & $\begin{array}{l}\text { Systematic and } \\
\text { perpendicular to } \\
\text { the femoral } \\
\text { mechanical axis }\end{array}$ & $\begin{array}{l}\text { Parallel to the distal } \\
\text { femoral joint line } \\
\text { (considering wear) }\end{array}$ & $\begin{array}{l}\text { Parallel to the distal } \\
\text { femoral joint line } \\
\text { (considering wear) }\end{array}$ & $\begin{array}{l}\text { Correct to }<5^{\circ} \text {, then } \\
\text { Parallel to the distal } \\
\text { femoral joint line } \\
\text { (considering wear) }\end{array}$ & $\begin{array}{l}\text { Parallel to the distal } \\
\text { femoral joint line } \\
\text { (considering wear) }\end{array}$ \\
\hline & & Target: $0^{\circ}$ & & & Target: $<5^{\circ}$ & Target: 0 to $5^{\circ}$ \\
\hline & Posterior cut & $\begin{array}{l}\text { External or neutral } \\
\text { rotation relative to } \\
\text { posterior condylar } \\
\text { line. } \\
\text { Measured resection or } \\
\text { gap-balancing } \\
\text { techniques. } \\
\text { Posterior or anterior } \\
\text { referencing } \\
\text { techniques }\end{array}$ & $\begin{array}{l}\text { Parallel to the } \\
\text { posterior condylar } \\
\text { line }\end{array}$ & $\begin{array}{l}\text { Parallel to the } \\
\text { posterior condylar } \\
\text { line }\end{array}$ & $\begin{array}{l}\text { Parallel to the } \\
\text { posterior condylar } \\
\text { line }\end{array}$ & $\begin{array}{l}\text { Surgical trans } \\
\text { epicondylar } \\
\text { axis; } \pm 3^{\circ}\end{array}$ \\
\hline & Mediolateral & Slightly lateralized & $\begin{array}{l}\text { Centered on } \\
\text { the notch }\end{array}$ & $\begin{array}{l}\text { Centered on } \\
\text { the notch }\end{array}$ & $\begin{array}{l}\text { Centered on } \\
\text { the notch }\end{array}$ & $\begin{array}{l}\text { Centered on } \\
\text { the distal femur }\end{array}$ \\
\hline \multirow[t]{4}{*}{$\begin{array}{l}\text { Tibial } \\
\text { component }\end{array}$} & Coronal cut & $\begin{array}{l}\text { Systematic and } \\
\text { perpendicular to } \\
\text { the tibial } \\
\text { mechanical axis }\end{array}$ & $\begin{array}{l}\text { Parallel to proximal } \\
\text { tibial joint line } \\
\text { (considering wear) }\end{array}$ & $\begin{array}{l}\text { Parallel to proximal } \\
\text { tibial joint line } \\
\text { (considering wear) } \\
\text { within safe zone of } \\
84^{\circ} \text { to } 92^{\circ}\end{array}$ & $\begin{array}{l}\text { Correct to }<5^{\circ} \text {, then } \\
\text { Parallel to proximal } \\
\text { tibial joint line } \\
\text { (considering wear) }\end{array}$ & $\begin{array}{l}\text { Perpendicular to the } \\
\text { tibial mechanical } \\
\text { axis }\end{array}$ \\
\hline & & Target: $0^{\circ}$ & Target: $-6^{\circ}$ to $9^{\circ}$ & Target: $-6^{\circ}$ to $2^{\circ}$ & Target: $<5^{\circ}$ & Target: $0 \pm 3^{\circ}$ \\
\hline & Slope & $\begin{array}{l}\text { Systematic. Between } \\
2^{\circ} \text { and } 7^{\circ} \text { relative to } \\
\text { sagittal tibial } \\
\text { mechanical axis }\end{array}$ & $\begin{array}{l}\text { Parallel to the medial } \\
\text { plateau slope }\end{array}$ & $\begin{array}{l}\text { Parallel to the medial } \\
\text { plateau slope }\end{array}$ & $\begin{array}{l}\text { Parallel to the medial } \\
\text { plateau slope }\end{array}$ & $\begin{array}{l}\text { Parallel to the medial } \\
\text { plateau slope; } \\
\text { Target: } 0^{\circ} \text { to } 3^{\circ}\end{array}$ \\
\hline & Rotation & $\begin{array}{l}\text { Towards the medial } \\
\text { third of the tibial } \\
\text { tuberosity }\end{array}$ & $\begin{array}{l}\text { Parallel to lateral } \\
\text { plateau long-axis }\end{array}$ & $\begin{array}{l}\text { Parallel to lateral } \\
\text { plateau long-axis }\end{array}$ & $\begin{array}{l}\text { Parallel to lateral } \\
\text { plateau long-axis }\end{array}$ & $\begin{array}{l}0 \text { to } 5^{\circ} \text { of external } \\
\text { rotation to Akagi's } \\
\text { line }\end{array}$ \\
\hline \multicolumn{2}{|c|}{$\begin{array}{c}\text { Knee } \\
\text { balancing }\end{array}$} & Soft tissues & Tibial cut & $\begin{array}{l}\text { Femoral cut (distal } \\
\text { and/or posterior) }\end{array}$ & $\begin{array}{l}\text { Tibial cut }+ \text { Soft } \\
\text { tissues }\end{array}$ & $\begin{array}{l}\text { Femoral and tibial } \\
\text { positioning }+ \text { Soft } \\
\text { tissues }\end{array}$ \\
\hline \multirow[t]{2}{*}{$\begin{array}{l}\text { Soft tissue } \\
\text { Release }\end{array}$} & $\begin{array}{l}\text { Femorotibial } \\
\text { joint }\end{array}$ & Frequent & None & None & Sometimes & Sometimes \\
\hline & $\begin{array}{l}\text { Lateral } \\
\quad \text { retinaculum }\end{array}$ & Sometimes & Rarely & Rarely & Rarely & Rarely \\
\hline \multicolumn{2}{|l|}{ Technologies } & All & All & Robotic-assisted & All & Robotic-assisted \\
\hline
\end{tabular}

adjustment of femoral and tibial cuts, implant positioning, or tissue release in three planes, of one or two degrees, to obtain optimal functional alignment. The precision offered by robotic assistance may make achieving non-neutral alignment targets more reproducible [41], reducing the risk of missing the target and producing significant outliers of the limb alignment. Theoretically, functional TKA reduces the need for periarticular soft-tissue releases if not desired by the surgeon while restoring the patient's native knee kinematics

\section{Surgical technique}

Robotic-assisted systems are constantly evolving both in hardware and software platforms and algorithms. Planning may initially begin preoperatively on a $3 \mathrm{D}$ and be completed during the surgery prior to bone cuts. Once the bone cuts have been made and the trial is in place the robotic system, soft tissue sensor or surgeon may discover a soft tissue imbalance.
Adjustments can then be assessed with software 3D manipulation virtually and then recut guidance or releases performed with the robotic-assisted system if indicated.

In the coronal plane, femoral component positioning is modified from a starting point of $0^{\circ}$ to the mechanical axis to balance the extension gap. In the sagittal plane, the femoral component is positioned to optimize the component sizing and to avoid femoral notch by flexing up to $5^{\circ}$. In the axial plane, the femoral implant is aligned to the transepicondylar axis with $3^{\circ}$ of freedom to balance the flexion gap. The size of the femoral component is selected using posterior referencing with the smallest size that does not overhang the femur, notch the anterior femur, or overhang mediolateral bone edges, and avoids overstuffing the patellofemoral joint. The femoral component is positioned at the center of the mediolateral cortical bone edges, with a small lateral position if necessary. In the coronal plane, the tibial component position is aligned to the tibial mechanical axis and modified to balance extension and 
flexion gaps by up to $3^{\circ}$ of varus. Valgus tibial position should be avoided. In the sagittal plane, the tibial component position is set to match the patient's pre-arthritic posterior tibial slope, modified to balance the flexion gap if necessary. In the axial plane, the tibial component is positioned using the line of Akagi.

The aim of functional alignment is to position the implants in the position that least compromises the knee ligaments envelope in 3D and hence to restore the obliquity and plane of the joint to that which the ligaments dictate. If the deformities are fixed, the soft-tissues release is required to balance the gaps, although the extent and frequency of such releases are smaller when compared with the MA technique.

\section{Results}

Several studies assessed the accuracy and the reproducibility of robotic-assisted surgery [42-46]. Sires and Wilson performed CT scans postoperatively to assess the precision of the image-based robotic-assisted TKA and found that $93 \%$ of the surgical measurements were $\leq 3^{\circ}$ of the CT measures postoperatively [46]. The use of preoperative CT scanning and the planning accuracy of robotic-assisted TKA resulted in well-balanced knees [47]. Nevertheless, no study has assessed the functional and clinical outcomes of this alignment technique, nor the implant survivorship.

\section{Conclusion}

Several concepts and evolving surgical techniques continue to develop personalized alignment in TKA. Personalized alignment aims to restore native knee alignment and improve functional outcomes after TKA. New technologies have increased the ability to restore native knee kinematics with TKA. A long-term follow-up is crucial to determine clinical outcomes and implant survivorship of these current alignment concepts.

\section{Conflict of interest}

SL: Consultant for Stryker, Smith Nephew, Heraeus, Depuy Synthes; Institutional research support from Groupe Lepine, Amplitude; Editorial Board for Journal of Bone and Joint Surgery (Am).

ESM, CB: declare that they have no conflict of interest.

CF: Consultant for Zimmer Biomet.

ES: Consultant for Corin.

SP: Royalties for Zimmer Biomet and Newclip; Consultant for Zimmer Biomet; Treasurer for European Knee Society.

\section{References}

1. Riviere C, Iranpour F, Auvinet E, et al. (2017) Alignment options for total knee arthroplasty: A systematic review. Orthop Traumatol Surg Res 103(7), 1047-1056.

2. Ritter MA, Faris PM, Keating EM, Meding JB (1994) Postoperative alignment of total knee replacement. Its effect on survival. Clin Orthop Relat Res 299, 153-156.
3. Riviere C, Iranpour F, Auvinet E, et al. (2017) Mechanical alignment technique for TKA: Are there intrinsic technical limitations? Orthop Traumatol Surg Res 103(7), 1057-1067.

4. Tew M, Waugh W (1985) Tibiofemoral alignment and the results of knee replacement. J Bone Joint Surg Br 67(4), 551-556.

5. Berend ME, Ritter MA, Meding JB, et al. (2004) Tibial component failure mechanisms in total knee arthroplasty. Clin Orthop Relat Res 428, 26-34.

6. Bonnin MP, Basiglini L, Archbold HA (2011) What are the factors of residual pain after uncomplicated TKA? Knee Surg Sports Traumatol Arthrosc 19(9), 1411-1417.

7. Forsythe ME, Dunbar MJ, Hennigar AW, Sullivan MJ, Gross M (2008) Prospective relation between catastrophizing and residual pain following knee arthroplasty: two-year follow-up. Pain Res Manag 13(4), 335-341.

8. Nashi N, Hong CC, Krishna L (2015) Residual knee pain and functional outcome following total knee arthroplasty in osteoarthritic patients. Knee Surg Sports Traumatol Arthrosc 23(6), 1841-1847.

9. Moser LB, Hess S, Amsler F, Behrend H, Hirschmann MT (2019) Native non-osteoarthritic knees have a highly variable coronal alignment: A systematic review. Knee Surg Sports Traumatol Arthrosc 27(5), 1359-1367.

10. Hovinga KR, Lerner AL (2009) Anatomic variations between Japanese and Caucasian populations in the healthy young adult knee joint. J Orthop Res 27(9), 1191-1196.

11. Khattak MJ, Umer M, Davis ET, Habib M, Ahmed M (2010) Lower-limb alignment and posterior tibial slope in Pakistanis: A radiographic study. J Orthop Surg (Hong Kong) 18(1), 22-25.

12. Hess S, Moser LB, Amsler F, Behrend H, Hirschmann MT (2019) Highly variable coronal tibial and femoral alignment in osteoarthritic knees: A systematic review. Knee Surg Sports Traumatol Arthrosc 27(5), 1368-1377.

13. Bellemans J, Colyn W, Vandenneucker H, Victor J (2012) The Chitranjan Ranawat award: is neutral mechanical alignment normal for all patients? The concept of constitutional varus. Clin Orthop Relat Res 470(1), 45-53.

14. Hirschmann MT, Hess S, Behrend H, Amsler F, Leclercq V, Moser LB (2019) Phenotyping of hip-knee-ankle angle in young non-osteoarthritic knees provides better understanding of native alignment variability. Knee Surg Sports Traumatol Arthrosc 27(5), 1378-1384.

15. Hirschmann MT, Moser LB, Amsler F, Behrend H, Leclercq V, Hess S (2019) Phenotyping the knee in young non-osteoarthritic knees shows a wide distribution of femoral and tibial coronal alignment. Knee Surg Sports Traumatol Arthrosc 27(5), 1385-1393.

16. Hirschmann MT, Moser LB, Amsler F, Behrend H, Leclerq V, Hess S (2019) Functional knee phenotypes: A novel classification for phenotyping the coronal lower limb alignment based on the native alignment in young non-osteoarthritic patients. Knee Surg Sports Traumatol Arthrosc 27(5), 1394-1402.

17. Hungerford DS, Krackow KA (1985) Total joint arthroplasty of the knee. Clin Orthop Relat Res 192, 23-33.

18. Nedopil AJ, Singh AK, Howell SM, Hull ML (2018) Does calipered kinematically aligned TKA restore native left to right symmetry of the lower limb and improve function? J Arthroplasty 33(2), 398-406.

19. Howell SM, Papadopoulos S, Kuznik KT, Hull ML (2013) Accurate alignment and high function after kinematically aligned TKA performed with generic instruments. Knee Surg Sports Traumatol Arthrosc 21(10), 2271-2280. 
20. Riviere C, Lazic S, Boughton O, Wiart Y, Villet L, Cobb J (2018) Current concepts for aligning knee implants: patientspecific or systematic? EFORT Open Rev 3(1), 1-6.

21. Gu Y, Roth JD, Howell SM, Hull ML (2014) How frequently do four methods for mechanically aligning a total knee arthroplasty cause collateral ligament imbalance and change alignment from normal in white patients? AAOS exhibit selection. J Bone Joint Surg Am 96(12), e101.

22. Howell SM, Howell SJ, Kuznik KT, Cohen J, Hull ML (2013) Does a kinematically aligned total knee arthroplasty restore function without failure regardless of alignment category? Clin Orthop Relat Res 471(3), 1000-1007.

23. Howell SM, Shelton TJ, Hull ML (2018) Implant survival and function ten years after kinematically aligned total knee arthroplasty. J Arthroplasty 33(12), 3678-3684.

24. Lee YS, Howell SM, Won YY, et al. (2017) Kinematic alignment is a possible alternative to mechanical alignment in total knee arthroplasty. Knee Surg Sports Traumatol Arthrosc 25(11), 3467-3479.

25. Sappey-Marinier E, Pauvert A, Batailler C, et al. (2020) Kinematic versus mechanical alignment for primary total knee arthroplasty with minimum 2 years follow-up: A systematic review. SICOT J 6, 18. https://doi.org/10.1051/sicotj/2020014.

26. Young SW, Sullivan NPT, Walker ML, Holland S, Bayan A, Farrington B (2020) No difference in 5-year clinical or radiographic outcomes between kinematic and mechanical alignment in TKA: A randomized controlled trial. Clin Orthop Relat Res 478(6), 1271-1279.

27. Yeo JH, Seon JK, Lee DH, Song EK (2019) No difference in outcomes and gait analysis between mechanical and kinematic knee alignment methods using robotic total knee arthroplasty. Knee Surg Sports Traumatol Arthrosc 27(4), 1142-1147.

28. Laende EK, Richardson CG, Dunbar MJ (2019) A randomized controlled trial of tibial component migration with kinematic alignment using patient-specific instrumentation versus mechanical alignment using computer-assisted surgery in total knee arthroplasty. Bone Joint J 101-B(8), 929-940.

29. McEwen PJ, Dlaska CE, Jovanovic IA, Doma K, Brandon BJ (2020) Computer-assisted kinematic and mechanical axis Total Knee Arthroplasty: A prospective randomized controlled trial of bilateral simultaneous surgery. J Arthroplasty 35(2), 443-450.

30. Dossett HG, Estrada NA, Swartz GJ, LeFevre GW, Kwasman BG (2014) A randomised controlled trial of kinematically and mechanically aligned total knee replacements: two-year clinical results. Bone Joint J 96-B(7), 907-913.

31. Shelton TJ, Gill M, Athwal G, Howell SM, Hull ML (2019) Outcomes in patients with a calipered kinematically aligned TKA that already had a contralateral mechanically aligned TKA. J Knee Surg 34(1), 87-93.

32. Sappey-Marinier E, White N, Gaillard R, et al. (2019) Increased valgus laxity in flexion with greater tibial resection depth following total knee arthroplasty. Knee Surg Sports Traumatol Arthrosc 27(5), 1450-1455.

33. Fang DM, Ritter MA, Davis KE (2009) Coronal alignment in total knee arthroplasty: Just how important is it? J Arthroplasty 24(6 Suppl), 39-43.
34. Winnock de Grave P, Luyckx T, Claeys K, et al. (2020) Higher satisfaction after total knee arthroplasty using restricted inverse kinematic alignment compared to adjusted mechanical alignment. Knee Surg Sports Traumatol Arthrosc. https://doi.org/ 10.1007/s00167-020-06165-4.

35. Nakamura S, Tian Y, Tanaka Y, et al. (2017) The effects of kinematically aligned total knee arthroplasty on stress at the medial tibia: A case study for varus knee. Bone Joint Res 6(1), 43-51.

36. Almaawi AM, Hutt JRB, Masse V, Lavigne M, Vendittoli PA (2017) The Impact of Mechanical and Restricted Kinematic Alignment on Knee Anatomy in Total Knee Arthroplasty. J Arthroplasty 32(7), 2133-2140.

37. Blakeney W, Beaulieu Y, Kiss MO, Riviere C, Vendittoli PA (2019) Less gap imbalance with restricted kinematic alignment than with mechanically aligned total knee arthroplasty: simulations on 3-D bone models created from CT-scans. Acta Orthop 90(6), 602-609.

38. MacDessi SJ, Griffiths-Jones W, Chen DB, et al. (2020) Restoring the constitutional alignment with a restrictive kinematic protocol improves quantitative soft-tissue balance in total knee arthroplasty: A randomized controlled trial. Bone Joint $\mathbf{J}$ 102-B(1), 117-124.

39. Kayani B, Konan S, Tahmassebi J, Oussedik S, Moriarty PD, Haddad FS (2020) A prospective double-blinded randomised control trial comparing robotic arm-assisted functionally aligned total knee arthroplasty versus robotic arm-assisted mechanically aligned total knee arthroplasty. Trials 21(1), 194.

40. Karachalios T, Komnos GA (2020) Individualized surgery in primary total knee arthroplasty. EFORT Open Rev 5(10), 663-671.

41. Kayani B, Konan S, Pietrzak JRT, Huq SS, Tahmassebi J, Haddad FS (2018) The learning curve associated with roboticarm assisted unicompartmental knee arthroplasty: A prospective cohort study. Bone Joint J 100-B(8), 1033-1042.

42. Kayani B, Konan S, Huq SS, Tahmassebi J, Haddad FS (2019) Robotic-arm assisted total knee arthroplasty has a learning curve of seven cases for integration into the surgical workflow but no learning curve effect for accuracy of implant positioning. Knee Surg Sports Traumatol Arthrosc 27(4), 1132-1141.

43. Sultan AA, Samuel LT, Khlopas A, et al. (2019) Robotic-arm assisted total knee arthroplasty more accurately restored the posterior Condylar offset ratio and the Insall-Salvati index compared to the manual technique; A Cohort-matched study. Surg Technol Int 34, 409-413.

44. Marchand RC, Khlopas A, Sodhi N, et al. (2018) Difficult cases in robotic arm-assisted total knee arthroplasty: A case series. J Knee Surg 31(1), 27-37.

45. Marchand RC, Sodhi N, Khlopas A, et al. (2018) Coronal correction for severe deformity using robotic-assisted total knee arthroplasty. J Knee Surg 31(1), 2-5.

46. Sires JD, Wilson CJ (2020) CT Validation of Intraoperative Implant Position and Knee Alignment as Determined by the MAKO Total Knee Arthroplasty System. J Knee Surg. https://doi.org/10.1055/s-0040-1701447.

47. Marchand RC, Sodhi N, Bhowmik-Stoker M, et al. (2019) Does the robotic arm and preoperative CT planning help with 3D intraoperative total knee arthroplasty planning? J Knee Surg. 32(8), 742-749.

Cite this article as: Lustig S, Sappey-Marinier E, Fary C, Servien E, Parratte S \& Batailler C (2021) Personalized alignment in total knee arthroplasty: current concepts. SICOT-J 7, 19 